\title{
Análisis de calidad basado en la vida útil de los rodamientos SKF 6205-2RS, mediante un módulo de prueba de vida acelerada
}

\section{Analysis of Quality based on the life of bearings mounted on an accelerated life test module}

\author{
Wilson Alejandro Huilca Alvarez. ${ }^{1} \&$ Cesar Daniel Arregui Toro. ${ }^{2}$ \\ Recibido: 01-02-2020 / Revisado: 18-03-2020 /Aceptado: 13-04-2020/ Publicado: 08-05-2020
}

\begin{abstract}
.
DOI: https://doi.org/10.33262/concienciadigital.v3i2.1.1228

The bearings are elements used in the industries; whose failure implies a great impact on the production of the entire manufacturing company. Its operation is vital for electromechanical equipment with rotating elements, either on shafts or in the bearings of industrial machines. The present study proposes the implementation of a quality control by means of an np-type diagram to SKF 6204-2RSH bearings, which will sometimes be elevated to high loads in an accelerated life test module reducing their useful life and taking samples of determined quantities of bearings determining the amount of defects per lot analyzed, with the aim of knowing if it is possible to determine the quality control of these elements by the number of defects. The results achieved with reduced frequency in the useful life of the bearings, showing the defects that can be quantified and recorded in the $\mathrm{np}$ tables, to later be analyzed in the characteristic quality control chart. After carrying out the analysis of the control graph, prudent wear of the 10 lots analyzed was observed with the exception of the number 7 , which shows a lag of the central line and a surplus of the ranges, accessories along the lower line and exceeded respectively, leading the study to suppose that the bearings part of this lot could be rejected before its operation in the hypothetical case of entering production and manufacturing. It should be noted that in the present analysis there were lubrication factors that were not selected and that may be

\footnotetext{
${ }^{1}$ Escuela Superior Politécnica de Chimborazo, Facultad de Mecánica, Chimborazo, Ecuador wilson.huilca@espoch.edu.ec
${ }^{2}$ Escuela Superior Politécnica de Chimborazo, Facultad de Mecánica, Chimborazo, Ecuador cesar.arregui@espoch.edu.ec
} 
subject to future research focused on changes that may be surgical under changes in the conditions of the lubricant applied to the bearings.

Keywords: Bearings, quality control, accelerated life test module, useful life

\section{Resumen.}

Los rodamientos son elementos ampliamente usados en las industrias, cuyo fallo implica un gran impacto en la producción de toda la empresa de manufactura. Su funcionamiento es vital para equipos electromecánicos con elementos rotativos, ya sea en ejes o en las chumaceras de máquinas industriales. El presente estudio plantea la implementación de un control de calidad mediante un diagrama tipo np a los rodamientos SKF 6204-2RSH, los cuales serán sometidos a elevadas cargas en un módulo de prueba de vida acelerada reduciendo significativamente la vida útil de los mismos y tomar muestras de cantidades determinadas de rodamientos determinando la cantidad de defectuosos por lote analizado, con el objetico de conocer si es posible determinar el control de calidad de estos elementos por el número de defectos. Los resultados conseguidos mostraron una reducción significativamente en la vida útil de los rodamientos, mostrando defectos que pudieron ser cuantificados y registrados en las tablas por atributos np, para posteriormente ser analizados en la gráfica característica de control de calidad. Después de realizar el análisis de la gráfica de control se observó un desgaste prudente de los 10 lotes analizados a excepción del número 7, que se muestra un desfase de la línea central y un excedente de los rangos, determinados por la línea inferior y superior respectivamente, conduciendo el estudio a suponer que los rodamientos parte de este lote pudiesen se rechazados antes de su operación en el caso hipotético de ingresar a producción y manufactura. Cabe señalar que en el presente análisis hubo factores de lubricación que no fueron considerados y que puede ser objeto para futuras investigaciones enfocadas en los cambios que pueden surgir bajo cambios en las condiciones del lubricante aplicado a los rodamientos

Palabras claves: Rodamientos, calidad, módulo de prueba de vida acelerada, vida útil.

\section{Introducción.}

En la actualidad casi cualquier equipo dinámico usa rodamientos, es por eso que Las fallas en rodamientos es uno de los problemas más comunes en los generadores síncronos. Aproximadamente $40 \%$ de las fallas en las máquinas eléctricas rotativas. (Sin, Soong, Ertugrul, $2013)$

Es por esta razón que es de vital importancia el uso de rodamientos que cumplan con las características de funcionamiento adecuadas, ya que un fallo en los mismo puede parar la 
producción de una fábrica completamente, Entre las causas más probables de fallas en rodamientos se encuentran distribuidas: $36 \%$ del resultado de una lubricación inadecuada; $34 \%$ resultado de una operación inadecuada como pudiera ser: cargas dinámicas excesivas sobre el rodamiento, producto de una falla de diseño de la máquina o de la presencia de fuerzas externas como desbalance y desalineamiento; $14 \%$ se debe a la contaminación, incluyendo humedad; y cerca de $16 \%$ son defectos originados en el proceso de montaje, (Duluth, Matthews 2013)

Conociendo estas cifras es inevitable no tomar medidas al momento de la selección de rodamientos, es por eso que mediante la presente investigación se busca encontrar un método con el cual poder determinar el porcentaje de rodamientos defectuosos. (Budynas, Nisbett, Shigley 2015)

En una investigación desarrollada por fabricantes, se determinó que los rodamientos operan aproximadamente $80 \%$ de su vida útil libres de defectos y cuando una falla ocurre, generalmente se encuentran en alguna de las cuatro diferentes etapas de falla

Etapa 1: Se tiene una vibración con componentes de alta frecuencia (generalmente mayores a $5 \mathrm{kHz}$ ), producto de la generación de ondas de esfuerzo y de otros tipos, las que, a su vez, excitan frecuencias naturales del soporte y las pistas del rodamiento, así como del sensor con el cual se realiza la medición. En esta etapa la temperatura no se incrementa y las grietas no son visibles y con frecuencia ocurren debajo de la superficie de las pistas. En esta etapa aún no es necesario el cambio del rodamiento. (Norton 2013)

Etapa 2: Las grietas empiezan a ser visibles al ojo humano y el rodamiento presenta emisión acústica, además de que la temperatura en algunos casos se incrementa. En esta etapa aparecen componentes frecuenciales relacionadas con las frecuencias de falla de los rodamientos en la zona de frecuencia baja e intermedia y hay un incremento de ruido audible. (Nieto 2017)

Etapa 3: Conforme la grieta avanza, pueden aparecer gran cantidad de bandas laterales alrededor de las frecuencias de falla relacionadas con la velocidad de rotación y modulación entre las diferentes frecuencias de falla cuando la grieta se ha extendido a pistas y elementos rodantes. En esta etapa se hace necesario comenzar a programar el cambio del rodamiento.

Etapa 4: Se encuentra cerca de una falla catastrófica, la emisión acústica aumenta significativamente y se produce sobrecalentamiento. En esta etapa, la vibración en la región de alta frecuencia disminuye, crece la componente a la velocidad de rotación y las vibraciones tienden a ser aleatorias. En este momento, será necesario el cambio inmediato del rodamiento. (Norton, 2013 ).

Como un caso real se puede tomar como ejemplo un tupí de banco en una carpintería, debido a los imperiosos requerimientos de la industria, en los últimos tiempos se ha incrementado la búsqueda 
de técnicas que permitan diagnosticar el estado de los rodamientos. Dentro de toda industria es conocido que los rodamientos son elementos fundamentales dentro de los activos industriales más utilizados como lo son bandas, generadores, motores, etc. Es conocido tambn que son elementos críticos en la mayoría de procesos en los cuales son utilizados, por lo cual es necesario y fundamental una lubricación óptima para que su ciclo de vida útil de trabajo sea lo más extensa posible y evitar los fallos antes de tiempo como son fatiga, vibraciones, desbalance o desalineación.

"Una herramienta eléctrica que se usa para fresar y agujerear un área determinada de piezas duras como la madera o el plástico. En la actualidad, el tupi, es una máquina muy habitual en los talleres gracias a su versatilidad que permite diversas operaciones como el galceado, el moldurado y el pulido de molduras. Se compone por una mesa con un sistema rotativo con fresa para realizar perfiles en la madera". (Martínez, Yoza 2019)

"Las máquinas han sido automatizadas en gran medida y los procesos han mejorado, pero la máquina tupí sigue conservando su funcionamiento tradicional que es el de realizar molduras, espigas, etc., es decir, fresar" (Martínez, Yoza 2019)

Otro factor importante al momento de determinar la vida útil de los rodamientos es la tribología misma que es la ciencia que estudia la lubricación, desgaste y fricción de dos cuerpos sólidos en movimiento relativo, (Aguilera 2010). Los fenómenos tribológicos básicos: fricción, desgaste y lubricación: todos están relacionados con la confiabilidad de los equipos, (Holmberg, 2001) en otros casos las fallas en rodamientos se basan en el empleo de registros de señales de vibración que juega en la actualidad un papel importante en el mantenimiento para analizar su comportamiento, (Medrano, Tello, Sarduy, Vera 2016).

Los rodamientos al ser uno de los componentes más críticos, son elementos que se usan ampliamente para evaluar el estado de una máquina rotativa. Cualquier falla inesperada del rodamiento desviaría el sistema mecánico del estado normal, como la pérdida de precisión, la reducción de la productividad e incluso el aumento de los riesgos de seguridad. (Yuanhang Chen, 2019) Más del $90 \%$ del número total de rodamientos están lubricados con grasa. El resto está lubricado con aceite. Las grasas lubricantes son coloides altamente estructurados, compuestos de aceite base, espesante y aditivos que mejoran el rendimiento. (Sathwik 2019)

Es por eso que actualmente los rodamientos son analizados mediante cálculos y análisis de vibraciones una vez que estos se encuentran en funcionamiento, por eso se ha decido estudiar el comportamiento de los rodamientos una vez que estos son fabricados y antes de que los mismos sean distribuidos, mediante las gráficas de control de calidad se busca sacar provecho de una de sus ventajas en el empleo de casos raros y en eventos con baja incidencia, en los cuales sería necesario obtener una muestra y un tiempo de seguimiento amplio, imposible en determinados 
casos, mediante métodos estadísticos convencionales. También por ello, son útiles para estudiar curvas de aprendizaje, la introducción de nuevas tecnologías y, en general, para valorar la calidad de los propios resultados asistenciales, porque su perfil es sensible a cambios de tendencias (positivas o negativas) muy sutiles en los resultados, que no se observarían con otros métodos. (Fortea 2019).

Las gráficas de control de calidad son ampliamente usadas para determinar el comportamiento de elementos en ciertos procesos, y variaciones de los mismos a través del tiempo sin que su calidad se vea afectada se utiliza graficas de control estadístico, para un mejor monitoreo del proceso (Devore 2008). El control estadístico de calidad aparece en la década de los 30 gracias a los laboratorios Bell, desarrollando con el tiempo técnicas eficaces para monitorear, evaluar, y poder observar el avance de la producción y mejorar las técnicas de calidad dentro de la empresa; reconociendo que con el tiempo se deberá dar variación a los procesos sin que estos afecten a la calidad del producto.

Teniendo como base los conceptos anteriores se desarrolló métodos estadísticos para establecer de una mejor forma los límites y graficas de control donde los resultados pudiesen ser monitoreados de una mejor manera; a la vez que Dodge y Roming avanzaban en la práctica del muestreo permitiéndonos inspeccionar el producto de una manera más veloz. Junto con sus teorías la necesidad de armamento de los EEUU en cantidades masivas en la segunda guerra mundial favoreció estos procesos ayudando a la aplicación con mayor amplitud de los conceptos previamente desarrollados (Garza 2007).

Con la información anterior podemos deducir que la calidad del producto depende exclusivamente del estado de los rodamientos al momento de realizar un análisis de calidad de los mismos, denotando que, en la industria, las máquinas y la producción dependen de los elementos constitutivos de ensambles y equipos industriales tales como los rodamientos.

$\mathrm{Al}$ acelerar la vida útil del rodamiento podemos tener un margen más amplio para su estudio, así como también para determinar en mayor medida los problemas que los mismos puedan tener antes de que estos sean distribuidos.

Un módulo de prueba de rodamientos, genera una aceleración de fallos, con el objetivo de cuantificar las características de vida útil del rodamiento en condiciones normales. Mediante el presente estudio, se podrá ejecutar un análisis de calidad de los rodamientos resultantes del módulo de prueba y de esta manera generar una predicción futura por lote. (González 2016). En nuestro caso particular el módulo de pruebas será un sistema de pulido eléctrico, mismo que es utilizado en microempresas convencionales, en este caso particular se utilizó el equipo de una carpintería aledaña. 
Figura 1 Diagrama del módulo de vida acelerada

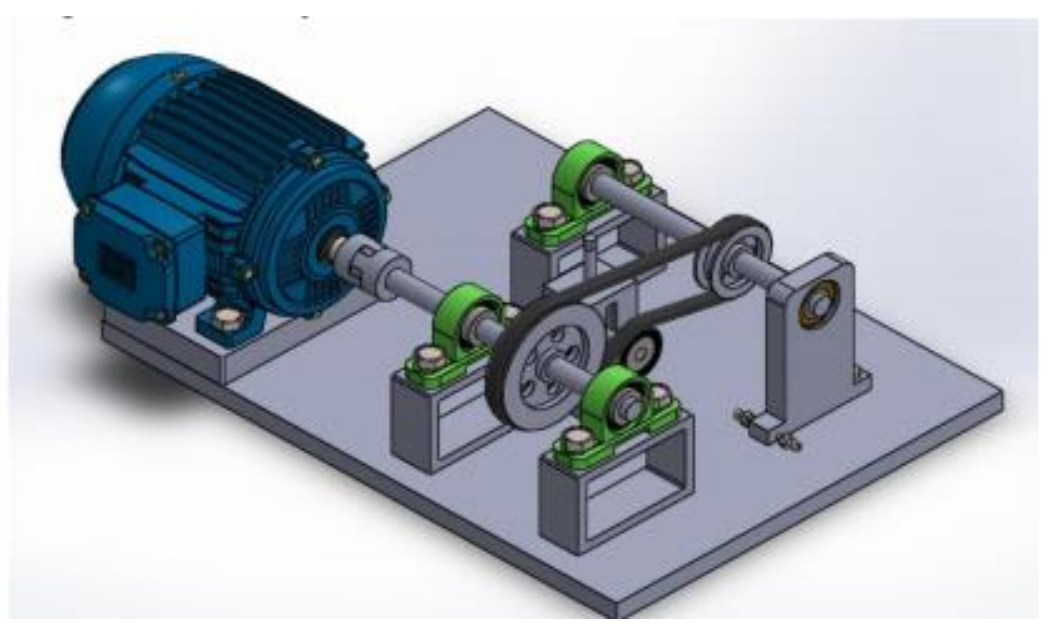

Fuente: Autónoma (gráfico en SolidWorks)

Mediante un análisis de control de calidad es posible determinar y desechar muestras defectuosas (Hernández, Fernández \& Baptista 2017). Por ende, al existir rodamientos defectuosos en una muestra se los puede clasificar y priorizar, antes de que estos sean usados en equipos rotativos, aumentando de esta forma la eficiencia de máquinas, evitando así, que los rodamientos con fallas de fábrica causen problemas en las empresas. Por tal motivo después de ser sometidos a nuestro módulo de prueba se debe realizar el análisis de calidad, verificando los defectos de cada rodamiento.

Sin embargo, hoy en día éstos análisis son muy extensos ya que se requiere evaluar a los rodamientos en condiciones de trabajo reales, por lo que en la mayoría de los casos determinar si un rodamiento se encuentra defectuoso antes de su adquisición es casi imposible. (Suarez, Omar \& Estupinan 2010).

El estudio de los rodamientos en esta investigación, se basa en un muestro tomado mediante un módulo de pruebas de vida acelera, para determinar de esta forma que lotes rechazar de acuerdo con la una vida útil promedio calculada.

Por lo tanto, se busca reducir la velocidad de desgaste de los rodamientos y analizar las fallas mecánicas causadas por el comportamiento del sistema de lubricación, para de esta manera conseguir la mantenibilidad de la vida útil del sistema. (Flores, Obed 2017)

Con el presente análisis y mediante diagramas de control de calidad se va a determinar de manera prematura rodamientos con defectos antes de que los mismos sean distribuidos, cuando éstos 
pierden energía cinética considerable por efectos de transmisión y elevadas cargas aplicadas. (Silva, Andrianoely, Manin, Ayasamy, Santini, Besnier, Remond, 2018).

\section{Metodología}

Para analizar el comportamiento de los rodamientos se les sometió a las siguientes condiciones de trabajo las cuales encontramos en una carpintería como alto nivel de partículas en suspensión en el ambiente (viruta de madera), sobrecargar al rodamiento por mal tensado de la banda que gira a la polea conducida la cual está conectada al rodamiento estudiado y también encontramos un alto contenido de virutas de madera en el lubricante del rodamiento.

El módulo de pruebas usado es el mostrado en la Figura 1, los elementos principales se encuentran montados en una placa base de acero AISI 1020 de $10 \mathrm{~mm}$ de espesor. La energía mecánica se suministra mediante un motor trifásico jaula de ardilla de $373 \mathrm{~W}$ y 4 polos cuyo eje conductor de acero inoxidable austenítico se encuentra acoplado a otro eje de similares características mismo en el cual se encuentra montada una polea de $125 \mathrm{~mm}$ que gira a una velocidad $1875 \mathrm{rpm}$ con una relación de transmisión de 1 a 2 .

La polea conducida de $64 \mathrm{~mm}$ acoplada a un tercer eje gira a una velocidad de $3750 \mathrm{rpm}$, la transmisión de movimiento entre poleas se lo realiza gracias a una banda de tipo correa en $\mathrm{V}$ de perfil AP24 pulgadas con doble cubierta e impregnada con neopreno.

Figura 2 Banco de pruebas de vida acelerada de rodamientos.

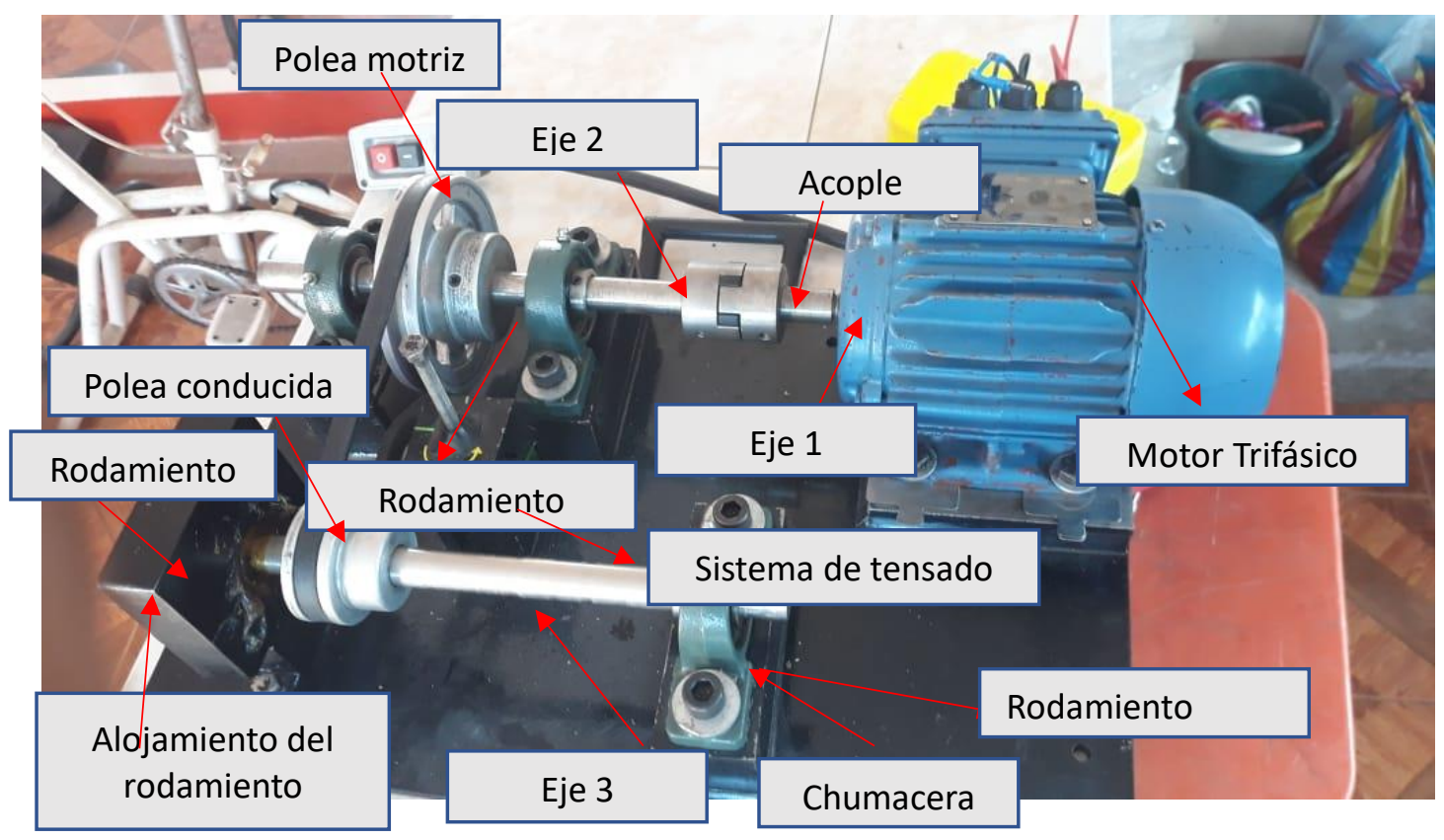

Fuente: Autónoma 
Las chumaceras son elegidas después de elegir el diámetro del eje, este procedimiento tomó tiempo ya que las chumaceras deben ser acordes al tamaño del eje y de los rodamientos que serán parte del estudio. Las chumaceras seleccionadas fueron las siguientes: marca CMB con designación HCP204 de la serie HC204,

El rodamiento que se utilizó fue el siguiente: rígido de bolas SKF 6205-2RS con las dimensiones que se detallan en la Figura 2, mismo que se encuentra montado en el módulo de pruebas como muestra la figura 1 , mismo que al girar a muy altas velocidades el daño del rodamiento se consigue en un tiempo muy reducido.

Tabla1. Dimensiones del rodamiento SKF 6205-2RS

\begin{tabular}{ccc}
\hline DIMENSION & VALOS NUMERICO & UNIDAD \\
\hline $\mathrm{d}$ & 25 & $\mathrm{~mm}$ \\
$\mathrm{D}$ & 52 & $\mathrm{~mm}$ \\
$\mathrm{~B}$ & 15 & $\mathrm{~mm}$ \\
peso & 0,13 & $\mathrm{~kg}$ \\
$\mathrm{cr}$ & 14,8 & $\mathrm{~N}$ \\
\hline
\end{tabular}

Fuente. Catálogo de rodamientos SKF

\section{Cálculo de la fuerza aplicada al rodamiento}

Para el cálculo de la fuerza se usarán dispositivos llamados galgas extensiométricas. Son sensores que generan resistencia de acuerdo la fuerza que se aplica, generando pulsos eléctricos medibles. Según la norma ISO 281 la duración de la vida nominal del rodamiento en horas es igual a la razón de una constante igual a 10 elevado a la sexta y 60 veces la velocidad de giro en rpm todo esto a su vez multiplicado por la razón entre la capacidad de carga dinámica base en $\mathrm{kN}$ y la carga dinámica equivalente del rodamiento en $\mathrm{kN}$ elevado a un exponente de ecuación de vida útil.

$$
L_{10 h}=\frac{10^{6}}{60 n}\left(\frac{C}{P}\right)^{p}
$$

Donde L10 es la vida nominal básica, $\mathrm{C}$ es la capacidad de carga dinámica básica, $\mathrm{P}$ es la carga dinámica equivalente de rodamiento, $\mathrm{p}$ es un exponente de la ecuación de la vida útil (3 para rodamientos de bolas y 10/3 para rodamientos de rodillos) y $\mathrm{n}$ es la velocidad de giro.

La capacidad de carga dinámica C la conseguimos de los catálogos de SKF de acuerdo con el tipo de rodamiento a utilizar. 
La carga dinámica equivalente P será el valor determinado mediante las galgas extensiométricas.

Para la investigación se ha decido utilizar un sistema de muestreo constante, con el módulo se busca simular un sistema de testeo para determinar si el rodamiento es adecuado para su distribución, si el rodamiento llegase a fallar antes del tiempo límite determinado, significará que el rodamiento no se encuentra en condiciones para trabajar y por ende el mismo deberá ser rechazado.

Es por eso que se ha decido utilizar las gráficas de control por tipo np, mismas que nos ayudarán a analizar el número de los defectuosos por subgrupo, el cual manifiesta que la línea central será igual al tamaño del lote multiplicado por la media de la proporción de defectos.

$$
L C=n \bar{p}
$$

El límite de control superior será igual al límite central más una constante igual a 3 que multiplica a la raíz cuadrada del límite central multiplicado a su vez con la diferencia entre 1 y la media de la proporción de defectuosos.

$$
L C S=L C+3 \sqrt{L C(1-\bar{p})}
$$

El límite de control inferior será igual al límite central menos una constante igual a 3 que multiplica a la raíz cuadrada del límite central multiplicado a su vez con la diferencia entre 1 y la media de la proporción de defectuosos.

$$
L C I=L C-3 \sqrt{L C(1-\bar{p})}
$$

Pará la gráfica serán usados los límites y se ubicarán los puntos con el número de rodamientos defectuosos en función del lote.

\section{Resultados}

Para determinar cuándo un rodamiento se encuentra defectuoso primero debemos calcular la vida útil del mismo cuando este se encuentra sometido a cargas de trabajo reales, analizando como base el comportamiento de los rodamientos bajo cargas de trabajo reales y registrando las medidas en la Tabla 1, tomando como primera medida, usando las galgas extensiométricas, al módulo sin ajuste del sistema de tensado, consiguiendo así una carga de trabajo dinámica equivalente $(\mathrm{P})$, las siguientes medidas fueron tomadas ajustando el sistema de tensado variando su distancia (D) cada $5 \mathrm{~mm}$ hasta llegar a los 50mm consiguiendo así la mayor carga de trabajo. 
Tabla 2 Carga dinámica equivalente en función de la distancia del módulo de tensado.

\begin{tabular}{cc}
\hline $\mathbf{D}(\mathbf{m m})$ & $\mathbf{P}(\mathbf{k N})$ \\
\hline 0 & 1,27 \\
5 & 2,54 \\
10 & 3,81 \\
15 & 5,08 \\
20 & 6,35 \\
25 & 7,62 \\
30 & 8,89 \\
35 & 10,16 \\
40 & 11,43 \\
45 & 12,7 \\
50 & 13,97 \\
\hline
\end{tabular}

Fuente:: Autónoma

Para determinar la capacidad de carga dinámica base nos ayudamos de los datos del rodamiento que se pueden conseguir en los manuales SKF como se detalla en la Figura 5.

Tabla 3 Capacidad de carga dinámica y estática para un rodamiento SKF 6204-2RSH Fuente. Autónoma

\begin{tabular}{llll}
\hline CAPACIDAD DE CARGA DINAMICA BASICA & $\mathrm{C}$ & 13,5 & $\mathrm{KN}$ \\
\hline CAPACIDAD DE CARGA ESTATICA BASICA & $\mathrm{Co}$ & 6,55 & $\mathrm{KN}$ \\
\hline
\end{tabular}

Fuente.: Autónoma

Tabla 4 Vida útil del rodamiento en horas en función de la carga.

\begin{tabular}{ccc}
\hline $\mathrm{D}(\mathrm{mm})$ & $\mathrm{P}(\mathrm{kN})$ & $(\mathrm{h})$ \\
\hline 0 & 1,27 & 5338 \\
5 & 2,54 & 667,27 \\
10 & 3,81 & 197,7 \\
15 & 5,08 & 83,4 \\
20 & 6,35 & 42,7 \\
25 & 7,62 & 24,71 \\
30 & 8,89 & 15,56 \\
35 & 10,16 & 10,42 \\
40 & 11,43 & 7,32
\end{tabular}




\begin{tabular}{ccc}
45 & 12,7 & 5,33 \\
50 & 13,97 & 4,01 \\
\hline
\end{tabular}

Fuente. Autónoma

Una vez tenemos la estimación de los datos sabemos que la vida útil promedio de un rodamiento en buen estado es de 5338 horas, gracias al módulo de pruebas de vida acelerada conocemos también que podemos reducir a 4,01 horas la vida útil de los rodamientos, con esta medida es mucho más fácil evaluar mediante diagramas de control de calidad el comportamiento de los rodamientos.

En la Tabla 3 se detallan los resultados obtenidos al realizar pruebas en 10 lotes $(\mathrm{N})$ con muestras de 45 rodamientos cada uno (n), mismos que serán sometidos a pruebas con la máxima distancia de tensado $50 \mathrm{~mm}$ para conseguir una carga de trabajo aproximada de $13,97 \mathrm{kN}$ esto debido a que realizar pruebas en rodamientos en condiciones normales tomaría 5338 horas por rodamiento lo que haría imposible un análisis de control de calidad, considerando así que un rodamiento se encuentra en perfecto funcionamiento si éste llega o supera las 4,01 horas de trabajo, en caso contrario será considerado como defectuoso (D).

Tabla 5 Rodamientos defectuosos encontrados en cada muestra.

\begin{tabular}{ccc}
\hline LOTE & TAMAÑO DEL LOTE & DEFECTUOSOS \\
\hline $\mathrm{N}$ & $\mathrm{n}$ & $\mathrm{D}$ \\
1 & 45 & 7 \\
2 & 45 & 9 \\
3 & 45 & 8 \\
4 & 45 & 10 \\
5 & 45 & 9 \\
6 & 45 & 11 \\
7 & 45 & 20 \\
8 & 45 & 11 \\
9 & 45 & 8 \\
10 & 45 & 9 \\
\hline
\end{tabular}

Fuente. Autónoma

Una vez tenemos los datos del muestreo el siguiente paso es realizar los cálculos para determinar la gráfica de control de calidad tipo np, se empieza por determinar la porción de defectos ( $\mathrm{Pi}$ ), misma que será igual a la división entre el número de rodamientos defectuosos (D) con el tamaño del lote (n) que en este caso es constante, los valores se detallan en la Tabla 4. 
Tabla 6 Rodamientos defectuosos encontrados en cada muestra.

\section{LOTE $\begin{gathered}\text { TAMAÑO } \\ \text { DEL LOTE }\end{gathered} \quad$ DEFECTUOSOS $\begin{gathered}\text { PROPORCIÓN } \\ \text { DE DEFECTOS }\end{gathered}$}

\begin{tabular}{cccc}
\hline $\mathrm{N}$ & $\mathrm{n}$ & $\mathrm{D}$ & $\mathrm{Pi}$ \\
1 & 45 & 7 & 0,16 \\
2 & 45 & 9 & 0,2 \\
3 & 45 & 8 & 0,18 \\
4 & 45 & 10 & 0,22 \\
5 & 45 & 9 & 0,2 \\
6 & 45 & 11 & 0,24 \\
7 & 45 & 20 & 0,44 \\
8 & 45 & 11 & 0,24 \\
9 & 45 & 8 & 0,18 \\
10 & 45 & 9 & 0,2 \\
\hline
\end{tabular}

Fuente. Autónoma

Al determinar la proporción defectos procedemos a calcular los límites de control central, superior e inferior, para el límite central usamos la ecuación (2) donde $\mathrm{n}$ es el tamaño del lote y es el promedio de la proporción de defectos $\mathrm{Pi}$, para determinar el límite de control superior e inferior usamos las ecuaciones (3) y (4) respectivamente, en donde LC es el límite central y es el promedio de la proporción de defectos $\mathrm{Pi}$.

$$
\begin{gathered}
L C=n \bar{p} \\
L C S=L C+3 \sqrt{L C(1-\bar{p})} \\
L C S=L C-3 \sqrt{L C(1-\bar{p})}
\end{gathered}
$$

Mediante la aplicación de estas fórmulas conseguimos los datos de la Tabla 5.

Tabla 7 Rodamientos defectuosos encontrados en cada muestra.

\begin{tabular}{cccc} 
PROMEDIO Pi & $\begin{array}{l}\text { LÍMITE CEN- } \\
\text { TRAL LC }\end{array}$ & $\begin{array}{l}\text { LÍMITE DE CONTROL LÍMITE } \\
\text { SUPERIOR LCS }\end{array}$ & $\begin{array}{l}\text { DE CON- } \\
\text { TROL INFERIOR LCI }\end{array}$ \\
\hline 0,23 & 10,2 & 18,63 & 1,77 \\
\hline
\end{tabular}

Fuente: Autónoma

Con los datos anteriores podemos elaborar la gráfica de control tipo np que se detalla en la Figura 
6, en la cual se colocarán el número de lotes $\mathrm{N}$ en el eje de las abscisas y el número de rodamientos defectuosos $\mathrm{D}$ en el eje de las ordenadas.

Tabla 8 Gráfico de control por atributos tipo np.

\begin{tabular}{|c|c|c|c|}
\hline $\begin{array}{l}\text { PROMEDIO } \\
\mathrm{Pi} \\
\quad \bar{p} \\
\end{array}$ & $\begin{array}{l}\text { LÍMITE } \\
\text { CENTRAL } \\
\text { LC }\end{array}$ & $\begin{array}{l}\text { LÍMITE DE CON- } \\
\text { TROL SUPERIOR } \\
\text { LCS }\end{array}$ & $\begin{array}{l}\text { LÍMITE DE CON- } \\
\text { TROL INFERIOR } \\
\text { LCI }\end{array}$ \\
\hline 0,23 & 10,20 & 18,63 & 1,77 \\
\hline
\end{tabular}

Fuente: Autónoma

Con los datos anteriores podemos elaborar la gráfica de control tipo np que se detalla en la Figura 6, en la cual se colocarán el número de lotes $\mathrm{N}$ en el eje de las abscisas y el número de rodamientos defectuosos $\mathrm{D}$ en el eje de las ordenadas.

Figura 3 Gráfico de control por atributos tipo np.

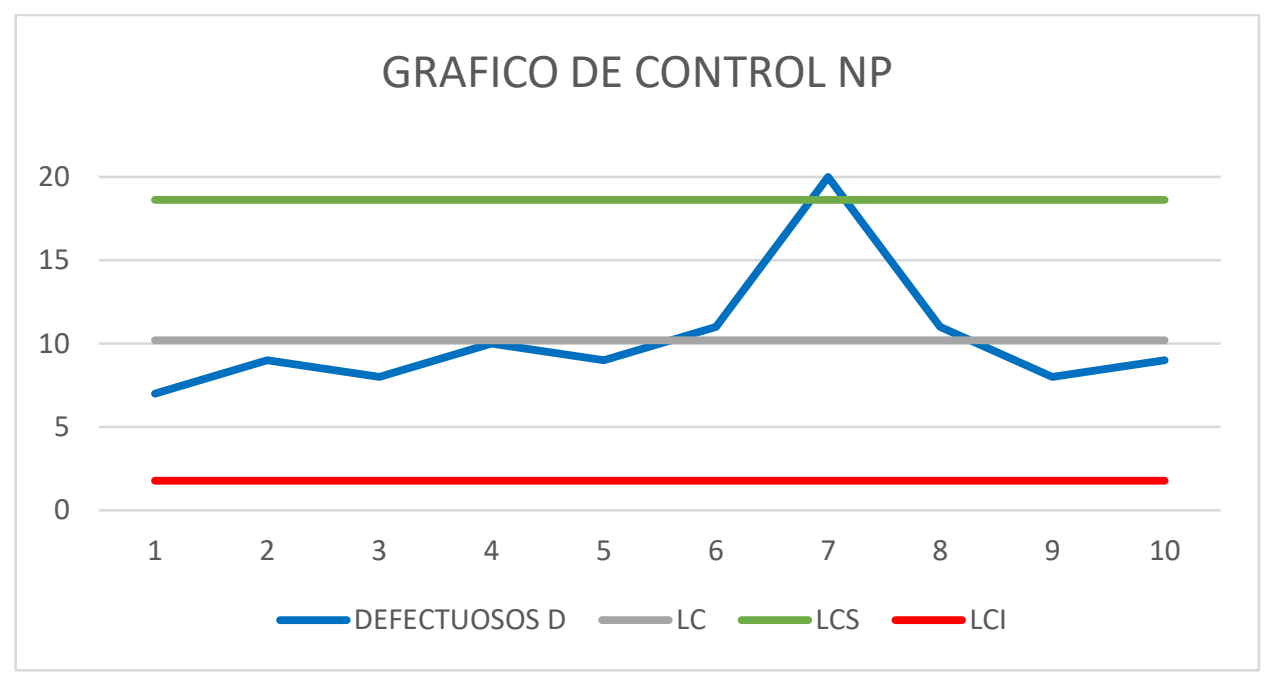

Fuente: Autónoma

\section{Discusión de resultados}

Con la ayuda de las tablas de SKF se puede apreciar un aproximado de la vida útil de los rodamientos en condiciones normales que como se pudo observar nos da un valor de 5338 horas, valor muy elevado en caso de querer realizar muestreos y gráficas de control de calidad, por tal razón se utilizó un módulo de vida acelerada para poder disminuir significativamente la vida útil del rodamiento, consiguiendo así que la evaluación de la calidad de los rodamientos sea mucho más rápida. 
El módulo de vida acelerada ayudo a observar el daño significativo que puede causar la falla de los rodamientos cuando están en funcionamiento en máquinas industriales tomando en cuenta que si $\mathrm{n}$ rodamiento falla afecta directamente a la función requerida del equipo y por lo tanto al paro del mismo

Como pudimos observar en la tabla 1, con la mayor carga el tiempo de vida del rodamiento se redujo a 4,01 horas logrando de esta manera, mayor eficacia al momento de realizar los muestreos, gracias a esto, la toma en los 10 lotes se pudo hacer en un tiempo aproximado de 3 meses.

Para este caso de estudio se decidió usar un diagrama de control tipo np, ya que fueron analizados 10 lotes, cada uno con una muestra de 45 rodamientos, y como se detalla en la Figura 4, se determinó el comportamiento de los rodamientos notando un excesivo incremento en el número de defectuosos (20), específicamente el lote número 7, denotando que dicho lote debería ser rechazado.

En la figura 4 se puede observar claramente en la gráfica de control de calidad que en el lote número 7 género un alto número de defectuosos por el cual se tomó la decisión de rechazar el lote completo, otros lotes que se tomaron en cuenta fueron el lote 6 y 8 que llegaron a generar un numero de defectuosos considerable pero no lo suficiente para rechazarlos. Este análisis se realiza considerando el comportamiento de las curvas, denotando que mientras más cercanas a la línea central estén, son más estables y menos desproporcionadas, y mientras más alejadas de la línea central, muestran desperfectos o problemas en la calidad de los rodamientos

Por cuestiones de diversos factores se decidió en el presente caso de estudio utilizar un lubricante multipropósito con las mismas características para cada rodamiento por lo cual todos lo rodamientos analizados llegaron a tener un tipo de desgaste característico por la utilización de este tipo de lubricante.

\section{Conclusiones.}

- Como se observa en las Tablas 1 y 2, existe una gran diferencia entre las cargas aplicadas al rodamiento a medida que se incrementa la distancia de aplicación del módulo de tensado, notando así una reducción de la vida útil de los rodamientos inversamente proporcional a la aplicación de carga.

- Al reducir considerablemente la vida útil de los rodamientos se puede conseguir un mayor margen de estudio al momento de realizar pruebas de calidad, esto debido a que se pueden evaluar muchos rodamientos en un tiempo muy corto permitiendo así sacar grandes muestras en varios lotes.

- De acuerdo con las observaciones plasmadas en la Figura 6, notamos que, para el presente caso de estudio, el lote número 7 sobrepasa el límite de control superior con un valor en la cantidad de rodamientos defectuosos muy elevado, por consecuente se decide rechazar dicho lote. 
- El lubricante es una parte importante al momento de realizar análisis de desgaste, en el presente artículo no se realizó dicho análisis ya que implicaría nuevos métodos de inspección y verificación, mismos que serán analizados para un próximo estudio

- El módulo de prueba fue probado y analizado, tomando en cuenta el ajuste de los ejes y todas las partes constitutivas. Todas las chumaceras estuvieron ubicadas de tal manera que el desgaste de los ejes sea el mismo en todas las partes del módulo de prueba

\section{Referencias bibliográficas.}

Aguilera Ramallo, R. (2010). Análisis de las pérdidas de potencia en rodamientos y poleas del sistema de corte de las KTP (Bachelor's thesis, Universidad de Holguín, Facultad de Ingeniería, Departamento de Ingeniería Mecánica.).

Budynas R, Nisbett K, Shigley J. (2015). Mechanical Engineering Design. Nueva York, Estados Unidos. McGraw-Hill Education. [Online]. Available: https://goo.gl/1TivVq

Fortea C. (2019). Técnicas de control de calidad en cirugía: Aplicación de las gráficas de control. Madrid, España. Cirugía Española, Volume 97, Pages 65-70.

Devore, J. L. (2008). Probabilidad y estadística para ingenierías y ciencias. Cengage Learning Editores.

Duluth G, Matthews P. (2013) Acoustic Fault Detection of Mechanical Systems with Active Noise Cancellation. Patent application number: 20130182865, recuperado de: http://www.faqs.org/patents/app/20130182865\#ixzz3SDtPXKxL.

Flores M. y Obed D. (2017) Análisis de desgastes mecánicos por tribología para reducir costos de mantenimiento del motor de tractor sobre orugas D6T-Caterpillar. Huancayo Perú, Iniversidad Nacional del Centro de Perú

González Luna, B. V., \& Quispe Saca, R. C. (2016). Comparación de la efectividad de las técnicas de ultrasonido pasivo y análisis de vibraciones en la detección de fallas incipientes en rodamientos (Bachelor's thesis, Escuela Superior Politécnica de Chimborazo).

Garza González, M. (2007). Modelo de indicadores de calidad en el ciclo de vida de proyectos inmobiliarios. Universidad Politécnica de Catalunya.

Hernández-Sampieri, R., Fernández-Collado, R., \& Baptista-Lucio, P. (2017). Selección de la muestra.

Holmberg K. (2001). Reliability aspects of tribology. Tribology International. ResearchGate. vol. $34, n^{0} 12$, p. 2. 
Medrano Z, Tello C, Sarduy J, Vera M. (2016). Nueva metodología de diagnóstico de fallas en rodamientos en una máquina síncrona mediante el procesamiento de señales vibroacústicas empleando análisis de densidad de potencia. Ingeniería, Investigación y Tecnología Vol. 17, $\mathrm{n}^{\mathrm{o}}$ 1, p. 74. Recuperado de: http://www.scielo.org.mx/scielo.php?script=sci_arttext\&pid=S1405-77432016000100073

Martínez Tabarez, Y. V., \& Yoza Plúa, G. R. (2019). Implementación de sistemas administrativos y reestructuración de la planta del taller artesanal Dekormuebles en la ciudad de Guayaquil para el año 2018 (Bachelor's thesis, Universidad de Guayaquil Facultad de Ciencias Administrativas). • Norton R. (2013). Diseño de maquinaria: síntesis y análisis de máquinas y mecanismos. México DF. McGraw-Hill. [Online]. Available: https://goo.gl/fGK4tx • Nieto J. (2007). Elementos de máquinas. Área de Ingeniería Mecánica. Albacete España. Universidad de Castilla. [Online]. Available: https://goo.gl/4wkZpd

Sin M, Soong W, Ertugrul N (2003). Induction machine on-line condition monitoring and fault diagnosis-a survey. University of Adelaide. Recuperado de https://www.researchgate.net/publication/237105539_Induction_machine_online_condition_monitoring_and_fault_diagnosis_-_A_survey

Salinas E, Link J, Athanasiou K. (2019). The tribology of cartilage: Mechanisms, experimental techniques, and relevance to translational tissue engineering. Maryland, Estados Unidos. Clinical Biomechanics.

Sathwik C, Lugt P. (2020). Channeling behavior of lubricating greases in rolling bearings: Identification and characterization. Tribology International, 143, 106061.

Silva A, Andrianoely M, Manin L, Ayasamy S, Santini C, Besnier E, and Remond D (2018). Optimization of power losses in poly-v belt transmissions via genetic algorithm and dynamic programming," Mechanism and Machine Theory, vol. 128, pp. 169-190, 2018. Disponible en https://doi.org/10.1016/j. mechmachtheory.2018.05.016

Suarez, C., Omar, H., \& Estupiñán López, I. F. (2010). Detección De Fallas En Un Motor De Inducción Usando Análisis De Vibraciones Mecánicas Bajo Distintos Escenarios De Carga (Doctoral dissertation, Universidad Industrial de Santander, Escuela De Ing. Mechanical).

Yuanhang Ch, Gaoliang P, Zhiyu Zhu S. (2019). A novel deep learning method based on attention mechanism for bearing remaining useful life prediction. Applied Soft Computing ScinceDirect, Recuperado de: https://www.sciencedirect.com/science/article/pii/S1568494619307008.

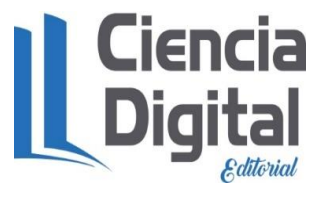




\section{PARA CITAR EL ARTÍCULO INDEXADO.}

Huilca Alvarez, W. A., \& Arregui Toro, C. D. (2020). Análisis de calidad basado en la vida útil de los rodamientos SKF 6205-2RS, mediante un módulo de prueba de vida acelerada. ConcienciaDigital, 3(2.1), 145-161. https://doi.org/10.33262/concienciadigital.v3i2.1.1228

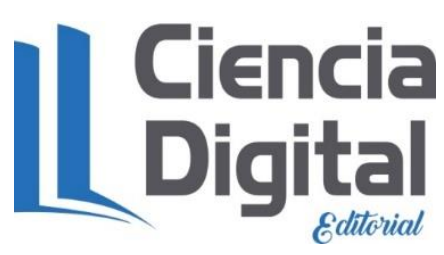

El artículo que se publica es de exclusiva responsabilidad de los autores y no necesariamente reflejan el pensamiento de la Revista Conciencia Digital.

El artículo queda en propiedad de la revista y, por tanto, su publicación parcial y/o total en otro medio tiene que ser autorizado por el director de la Revista Conciencia Digital.
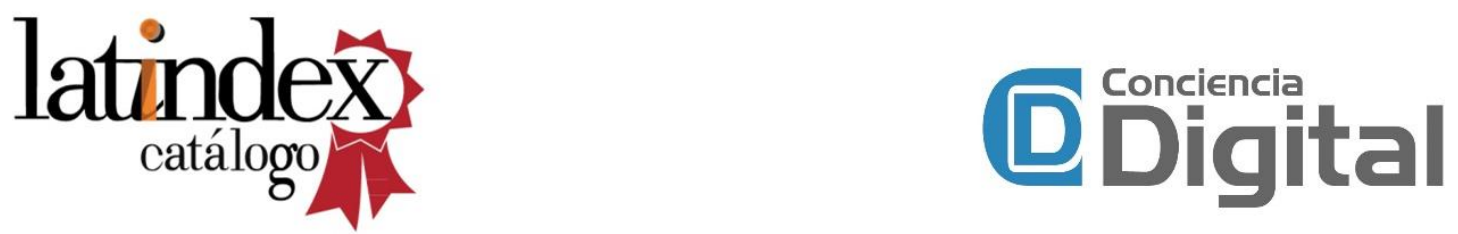